\title{
LOS CENTROS ESPECIALES DE EMPLEO EN CASTILLAY LEÓN, 2007-2013: EFECTOS DE LA CRISIS EN FUNCIÓN DE LA PERSONALIDAD JURÍDICA QUE ADOPTAN
}

\author{
POR \\ María Isabel MANZANO MARTÍN ${ }^{1}$ \\ María Nieves REDONDO MARTÍN ${ }^{2}$ \\ María Paz ROBLES PARAMIO ${ }^{3}$
}

\section{RESUMEN}

Este trabajo surge con el propósito de realizar un estudio, económico-financiero, sobre los Centros Especiales de Empleo localizados en Castilla y León, a partir de una clasificación en función de la personalidad jurídica que adoptan y comprobar cómo les puede afectar la crisis económica iniciada a finales de 2007. Se analizan diferentes partidas recogidas en sus estados financieros y se comparan los resultados del análisis con los obtenidos en años anteriores, periodo 2007-2013, al objeto de mostrar una perspectiva más amplia de su tamaño, desarrollo, crecimiento y comportamiento. Para ello se utilizan las siguientes magnitudes económicas: activo total, cifra de negocios y resultados. Se compara la variable empleo con las subvenciones recibidas por los Centros poniendo de manifiesto como la crisis sí les afecta en función de la personalidad jurídica que adoptan, siendo las entidades más perjudicadas las asociaciones y personas físicas, pudiendo incluso ocasionar su desaparición. Además se introduce la cuenta de reversión para los Centros, que mide qué porcentaje de las ayudas públicas recibidas es devuelto a la sociedad.

Palabras clave: Centro Especial de Empleo, personalidad jurídica, análisis económicofinanciero, regional, ayudas de la administración pública.

\footnotetext{
${ }^{1}$ Profesora del Departamento de Economía Financiera y Contabilidad. Universidad de Valladolid. Dirección de correo electrónico: imanzano@eco.uva.es.

${ }^{2}$ Profesora del Departamento de Economía Financiera y Contabilidad. Universidad de Valladolid. Dirección de correo electrónico: nieves@eco.uva.es.

${ }^{3}$ Profesora del Departamento de Economía Financiera y Contabilidad. Universidad de Valladolid. Dirección de correo electrónico: mariapaz@eco.uva.es.

REVESCO N 122 - Tercer Cuatrimestre 2016 - ISSN: 1885-8031 - www.ucm.es/info/revesco

http://dx.doi.org/10.5209/REVE.53241

Fecha de recepción: 13/10/2015

Fecha de aceptación: 18/03/2016
} 
Claves ECONLIT: G00; M41; P25.

\title{
SPECIAL EMPLOYMENT CENTERS IN CASTILE AND LEÓN 2007-2013: EFFECTS OF THE CRISIS IN RELATION TO THESE CENTERS' REGISTERED LEGAL PERSONALITIES
}

\begin{abstract}
The aim of this paper is to carry out an economic and financial study of the Special Employment Centres in Castile and León based on a classification of these entities' registered legal personalities in order to view how the economic crisis that began at the end of 2007 may have affected them. Various items from the Centres' financial statements are analysed and the results are compared to those from the period 2007-2013 as to provide a broader perspective of their size, development, growth and behaviour. The following economic figures were used: total assets, turnover and revenue. The variable "employment" is compared with the subsidies received by the Centres, showing that the crisis does affect the Centres depending on their registered legal personalities. Associations and physical persons are the most affected personalities, to the point of possible extinction. An account reversal for the Centres is also included in this article, which measures the percentage of public aid received by the Centres that is returned to society.
\end{abstract}

Keywords: Special Employment Center, legal personality, economic and financial analysis, regional, public administration aid.

\section{INTRODUCCIÓN}

Este trabajo surge con el propósito de realizar un estudio, económico-financiero, sobre los Centros Especiales de Empleo (CEdE) localizados en Castilla y León, a partir de una clasificación en función de la personalidad jurídica que adoptan y comprobar cómo les puede afectar la crisis económica iniciada a finales de 2007.

La forma jurídica que presentan los CEdE, tiene importantes repercusiones, tanto para la creación como para su desarrollo posterior, pudiendo influir en la conducta de estas entidades y en su actuación económica, debido a las implicaciones económicas, fiscales y legales que contiene. 
La Ley 13/1982, de 7 de abril, de Integración Social de los Minusválidos (LISMI), derogada por el vigente Real Decreto Legislativo 1/2013 (RDL 1/2013), de 29 de noviembre, por el que se aprueba el Texto Refundido de la Ley General de derechos de las personas con discapacidad y de su inclusión social, recogía por primera vez la existencia de los CEdE y establecía en su Art. 41 que «las personas con discapacidad que no puedan ejercer una actividad laboral en las condiciones habituales, podrán ser empleadas en los Centros Especiales de Empleo».

Los $\mathrm{CEdE}^{4}$ son concebidos como un medio para asegurar un empleo remunerado a las personas con discapacidad, en adelante PCD, además de proporcionar a estas personas el acceso al régimen de empleo ordinario.

A pesar de que al menos el $70 \%$ de la plantilla ${ }^{5}$ de estos Centros tiene que estar formada por trabajadores con un grado de discapacidad superior al 33\%, esta condición ${ }^{6}$ no ha supuesto un obstáculo en el desarrollo y crecimiento de los Centros. Éstos han demostrado ser capaces de realizar cualquier tipo de actividad y de operar en el mercado de bienes y servicios como cualquier otra modalidad de empresa. Sin embargo, estas entidades son muy poco conocidas por parte de la sociedad, e incluso en mucho casos se desconocen totalmente.

Conforme al Art. 45 del RDL 1/2013, los CEdE podrán ser «creados tanto por organismos públicos y privados como por las empresas, siempre con sujeción a las normas legales, reglamentarias y convencionales que regulen las condiciones de trabajo». No obstante, en Castilla y León el 100\% de los CEdE registrados tienen carácter privado, tal y como se desprende de los diferentes registros publicados por la Consejería de Economía y Empleo para todo el periodo de estudio, presentando una gran heterogeneidad en cuanto a dimensión, número de trabajadores empleados, personalidad jurídica y actividad que realizan.

\footnotetext{
${ }^{4}$ El Real Decreto Legislativo 1/2013 en su Art.43. de Centros especiales de empleo para la inclusión laboral de las personas con discapacidad, define a éstos como: «Los centros especiales de empleo son aquellos cuyo objetivo principal es el de realizar una actividad productiva de bienes o de servicios, participando regularmente en las operaciones del mercado, y tienen como finalidad el asegurar un empleo remunerado para las personas con discapacidad; a la vez que son un medio de inclusión del mayor número de estas personas en el régimen de empleo ordinario. Igualmente, los centros especiales de empleo deberán prestar, a través de las unidades de apoyo, los servicios de ajuste personal y social que requieran las personas trabajadoras con discapacidad, según sus circunstancias y conforme a lo que se determine reglamentariamente».

${ }^{5}$ Art. 43.2 del Real Decreto Legislativo 1/2013.

${ }^{6}$ Real Decreto 2273/1985, de 4 de diciembre, por el que se aprueba el Reglamento de los Centros Especiales de Empleo definidos en el artículo 42 de la Ley 13/1982, de 7 de abril, de Integración Social del Minusválido.
} 


\section{REVISIÓN DE LA LITERATURA}

Los estudios acerca de los aspectos económicos-financieros de los CEdE han sido mínimos. No obstante, hay que resaltar que en los últimos años los investigadores han dado cada vez más importancia al estudio de este tipo de entidades. Aun así, la mayoría de los estudios existentes se centran principalmente en aspectos jurídicos y sociales.

Uno de los primeros trabajos sobre los CEdE fue realizado por Del Río (1984) y promovido por la Unión Sindical de Madrid - Región de CCOO. El objetivo del mismo era realizar una radiografía sobre los CEdE, trasladar a la sociedad la información y la reflexión y aportar pautas para el diseño de políticas específicas dirigidas a mejorar las condiciones laborales y, en definitiva, la calidad de vida laboral de las personas con discapacidad.

Visier (1998) recoge la problemática que presentan las diversas concepciones del trabajo protegido, y analiza desde un punto de vista sociológico las condiciones de trabajo en los talleres y centros protegidos de varios países. Plantea la diversidad de los marcos jurídicos en los que se desarrollan estos Centros, que oscilan entre la normativa aplicada a una empresa ordinaria y la aplicada a los establecimientos de orientación terapéutica. Concluye el trabajo considerando que la discapacidad no constituye un obstáculo a la integración plena en el ejercicio profesional, pero sí sigue siendo indispensable la protección a los trabajadores para que éstos consigan su autonomía.

Tuset (2002) detalló en profundidad y en base a la normativa legal el concepto de CEdE, su naturaleza, clases, constitución y registro. Rubio (2003) realizó un estudio descriptivo acerca de la tasa de actividad para el colectivo de las personas con discapacidad y estableció una comparación entre dos años (1986 y 1999), en base a la Encuesta sobre Discapacidades y Estados de Salud (INE, 2000). En el estudio se confirma que en aquellos dos años la situación laboral de las personas con discapacidad, si se comparaba con la población en general, era negativa: los trabajadores con discapacidad mostraban bajas tasas de actividad y altas tasas de desempleo. El autor hace especial referencia a los CEdE como modalidad de empleo protegido. En el estudio de Gómez, Román y Rojo (2010) se recoge un análisis de la calidad del empleo generado por las sociedades laborales y por los CEdE y la vinculación positiva para la integración social en el ámbito territorial de la comunidad autónoma de Castilla y León. También es analizada la normativa laboral de los CEdE y los principales rasgos de su aplicación práctica por Jordán de Urries y Verdugo (2010). 
Calvo (2004) recoge la situación jurídica y las perspectivas de futuro de los CEdE, así como su importancia en el marco de la Economía Social al facilitar el desarrollo personal y social de las PCD en base a su integración en el mercado laboral y Carrio (2005) realiza un estudio descriptivo de los CEdE en el Principado de Asturias.

González (2008) presenta un estudio sobre la eficiencia y productividad de los CEdE, considerando que la incorporación de modelos matemáticos de eficiencia técnica podría ser una herramienta necesaria para medir la distribución de las diferentes ayudas públicas a los Centros.

El estudio elaborado por KPMG en el marco del Programa Operativo del FSE de Lucha contra la Discriminación 2007-2013 cofinanciado por el Fondo Social Europeo, recoge como objetivo el de medir la contribución de los CEdE a las Administraciones Públicas, concluyendo que los Centros retornan a la sociedad 1,44€ por cada euro que perciben de las ayudas públicas, mediante impuestos, tributos y aportaciones a la seguridad social.

Por último los trabajos de Rodríguez (2012), Redondo (2013) y Camacho, Gelashvili y Segovia (2015), ponen de relieve el interés reciente que han suscitado los CEdE, centrándose en su continuo crecimiento y supervivencia.

\section{METODOLOGÍA DEL ESTUDIO Y MUESTRA DE CENTROS ESPECIALES DE EMPLEO}

Se realiza una primera recopilación de información sobre los CEdE, lo que ha supuesto un primer conocimiento y contacto con este tipo de entidades.

La investigación que se lleva a cabo en este trabajo es principalmente de tipo cuantitativo relacionado con la práctica contable, lo que ha permitido la obtención de indicadores numéricos y comparables.

La base de datos en este estudio se elabora a partir del balance de situación, de la cuenta de pérdidas y ganancias y del informe obligatorio o memoria que debe recoger el cumplimiento de objetivos económicos y de ajuste personal y social de los diferentes Centros seleccionados en la muestra. La principal fuente de esta información son las memorias socioeconómicas que los CEdE están obligados a depositar en la Dirección General de Economía Social adscrita a la Consejería de Economía y Empleo de la Junta de Castilla y León (JCyL). 
Durante el tiempo que ha durado la selección de la muestra y la elaboración de la base de datos surgieron una serie de condicionantes, lo que ha hecho que no se hayan podido seleccionar todos los Centros que aparecen registrados en la comunidad de Castilla y León. Estos condicionantes han venido marcados principalmente por:

1.- La recepción de los documentos: las cuentas anuales de los CEdE para el periodo de estudio son facilitadas, como ya se ha comentado, por la Dirección General de Economía Social bajo el anonimato ${ }^{7}$, donde todos los datos de identificación personal (nombre comercial, dirección, relación nominal de trabajadores, etc.) habían sido ocultados, siendo prácticamente imposible determinar a qué empresa pertenecía la información.

2.- Existen empresas que en la actualidad prestan poca atención a la elaboración de las cuentas anuales y a la presentación de sus estados financieros. Sus estados presentan fallos tanto de forma como de contenido, lo que llevó a rechazarlas.

3.- Algunos Centros, principalmente de carácter fundacional, realizan actividades en más de una provincia de Castilla y León, e incluso fuera de la Comunidad, y presentan cuentas anuales consolidadas sin indicar qué cuentas pertenecen a cada Centro filial.

En el estudio, por tanto, no se recogen todos los CEdE de Castilla y León. Solo los Centros que han cumplido con los requisitos ${ }^{8}$ enumerados por la Asociación Española de Contabilidad y Administración de Empresas (AECA 1991), y que poseen datos completos. Así Somoza y Vallverdú (2003:7) consideran siguiendo la normativa del Plan General de Contabilidad (PGC, 2008) que «una empresa dispone de datos completos si deposita información contable íntegra en los últimos ejercicios anteriores en el Registro Mercantil, esto es, balance de situación a fecha de cierre del ejercicio, cuenta de pérdidas y ganancias, memoria e informe de auditoría». El hecho de que algunos de los Centros analizados no están obligados a presentar las cuentas en el Registro Mercantil dificultó poder contar con una muestra más amplia o incluso completa.

\footnotetext{
${ }^{7}$ Art. 8. Tratamiento de los datos del Registro: «El tratamiento de los datos de carácter personal contenidos en los expedientes que dan lugar a la calificación y posterior inscripción en el Registro quedará sujeto al régimen de especial protección previsto en la Ley Orgánica 15/1999, de 13 de diciembre, de protección de datos de carácter personal. El resto de los datos quedará sujeto a lo dispuesto en el artículo 37 de la Ley 30/1992, de 26 de noviembre, de Régimen Jurídico de las Administraciones Públicas y del Procedimiento Administrativo Común».

${ }^{8}$ AECA (1991, p: 23-26): Identificabilidad, oportunidad, claridad, relevancia, imparcialidad, objetividad, verificabilidad e integridad.
} 
Después de realizar un minucioso y profundo análisis, el número de Centros con información completa recogidos en la muestra se detalla en el Cuadro 1.

Cuadro 1. Centros especiales de empleo registrados en Castilla y León y muestra seleccionada

\begin{tabular}{|c|c|c|c|c|c|c|c||}
\hline CEdE & $\mathbf{2 0 0 7}$ & $\mathbf{2 0 0 8}$ & $\mathbf{2 0 0 9}$ & $\mathbf{2 0 1 0}$ & $\mathbf{2 0 1 1}$ & $\mathbf{2 0 1 2}$ & $\mathbf{2 0 1 3}$ \\
\hline \hline Población (Registro) & 158 & 161 & 165 & 173 & 185 & 190 & 192 \\
\hline \hline Muestra & 114 & 115 & 113 & 103 & 103 & 103 & 103 \\
\hline \hline
\end{tabular}

Fuente: Elaboración propia a partir del registro de CEdE de Castilla y León y de la documentación presentada por los Centros en la Dirección de Economía Social.

Se inicia el estudio analizando diferentes partidas recogidas en el balance, ya que este estado es el elemento principal del conjunto de los estados financieros y básico en el análisis, por la información cuantitativa que proporciona (Bernstein, 1984).

Amat (2008:43-44) establece que «el análisis del balance permite evaluar la situación de: liquidez, solvencia, endeudamiento, independencia financiera, garantía patrimonial frente a terceros, capitalización, eficiencia en la gestión de los activos que invierte la empresa, y equilibrio financiero».

Posteriormente, se realiza un análisis vertical y horizontal donde se aprecia la evolución del activo y pasivo para el periodo analizado (2007-2013). Se complementa el análisis con la utilización de estadísticos descriptivos de las magnitudes: activo, ingreso neto de la cifra de negocios y beneficio bruto, que permiten definir el tamaño, desarrollo, comportamiento y las características de este grupo de entidades.

A continuación, se detalla la evolución de variables significativas: empleo, gasto de personal y subvenciones para determinar los efectos de la crisis económica sobre los CEdE.

\subsection{Descripción de la población objetivo: Los Centros Especiales de Empleo atendiendo a su personalidad jurídica}

Con respecto a la personalidad jurídica que presentan los CEdE analizados de la Comunidad de Castilla y León, se observa en el Cuadro 2 de distribución que más del $80 \%$ de los Centros optan por una forma jurídica mercantil, siendo la sociedad limitada la más utilizada. Esto se debe a que ésta ofrece ciertas ventajas respecto al resto (el capital inicial es poco elevado, responsabilidad limitada y un control sencillo de la gestión administrativa). 
Cuadro 2. Distribución de los CEdE de Castilla y León atendiendo a la forma jurídica. (\%). Muestra

\begin{tabular}{||c|c|c|c|c|c|c|c||}
\cline { 2 - 8 } \multicolumn{1}{c|}{} & $\mathbf{2 0 0 7}$ & $\mathbf{2 0 0 8}$ & $\mathbf{2 0 0 9}$ & $\mathbf{2 0 1 0}$ & $\mathbf{2 0 1 1}$ & $\mathbf{2 0 1 2}$ & $\mathbf{2 0 1 3}$ \\
\hline \hline Asociación & 0,15 & 0,13 & 0,12 & 0,11 & 0,11 & 0,17 & 0,09 \\
\hline Cooperativa & & & & 0,01 & 0,01 & 0,01 & 0,01 \\
\hline Fundación & 0,03 & 0,03 & 0,03 & 0,03 & 0,03 & 0,03 & 0,03 \\
\hline Persona Física & 0,03 & 0,02 & 0,02 & 0,02 & 0,02 & 0,01 & 0,01 \\
\hline Sociedad Anónima & 0,14 & 0,14 & 0,14 & 0,14 & 0,13 & 0,13 & 0,13 \\
\hline Sociedad Limitada & 0,67 & 0,67 & 0,69 & 0,70 & 0,71 & 0,71 & 0,74 \\
\hline \hline Total General & 114 & 115 & 113 & 103 & 103 & 103 & 103 \\
\hline \hline
\end{tabular}

Fuente: Elaboración propia a partir del registro de CEdE de Castilla y León y de la documentación presentada por los Centros en la Dirección de Economía Social.

En contraposición están los Centros que son personas físicas jurídicamente. Éstos siguen el régimen de empresarios individuales, habitualmente conocidos como autónomos, lo que lleva un tratamiento fiscal más desfavorable e implica mayor responsabilidad por parte del empresario. Esta forma de personalidad tiende a desaparecer en el periodo estudiado.

En 2010 empieza a descender el número de asociaciones y empresarios individuales y se constituyen bien como cooperativas o como sociedades limitadas, estas últimas siguen siendo la figura mayoritaria ${ }^{9}$. En 2013 aumentan los Centros cuya forma jurídica es la de responsabilidad limitada hasta en un $74 \%$ del total (103 Centros), disminuyendo las otras personalidades (solo el 1\% presentaba la forma de empresario individual). Esta distribución se mantiene durante todo el periodo de estudio.

Es importante resaltar como a pesar de que en torno al $84 \%$ de los Centros estudiados en este trabajo presentan la forma de sociedades mercantiles, ninguno de ellos ha adoptado la forma de sociedad laboral. En la actualidad están registrados un total de 194 Centros en Castilla y León, entre los cuales tampoco se recogen organizaciones que adopten la forma de sociedad laboral (Registro de CEDE de la Junta de Castilla y León, actualizado a 1/10/2015).

\section{ANÁLISIS DEL TAMAÑO DE LOS CENTROS ESPECIALES DE EMPLEO}

A través de la distribución estadística de las magnitudes: activo agregado total, cifra de negocios y beneficio bruto, se realiza el análisis del tamaño de los CEdE por personalidad

\footnotetext{
${ }^{9}$ En 2007 las sociedades limitadas representaban el 67\% del total de la muestra seleccionada, el 15\% son asociaciones, el 14\% sociedades anónimas y las fundaciones y las personas físicas representan por igual un $5 \%$.
}

REVESCO No 122 - Tercer Cuatrimestre 2016 - ISSN: 1885-8031 - www.ucm.es/info/revesco 
jurídica (Anexos: 2-3-4). Se aporta la media, la mediana y los percentiles 75 y 25 como indicadores de la tendencia central, y la desviación típica como medida de la dispersión.

Rodríguez (2002: 16) recoge que «el análisis de la dimensión de las compañías es de gran relevancia pues el tamaño es un factor que condiciona de forma significativa los valores presentados por las magnitudes contables».

Para las asociaciones, fundaciones, sociedades anónimas y sociedades limitadas las medias son superiores a las medianas, mostrando una asimetría positiva. Esta tendencia se repite a lo largo de los años de estudio. Teniendo en cuenta el valor de la media en este grupo de empresas, se deduce que la dimensión de las asociaciones, fundaciones y sociedades limitadas son similares, pudiendo encuadrarlas como pequeñas empresas (activo menor de 10.000.000€). Destacan las cifras que corresponden a las sociedades anónimas, donde se observan valores superiores a otras formas jurídicas. En 2013, la media del activo para este tipo de entidades es de 22.101.746€, lo cual indica que se trata de empresas de tamaño mediano. Sin embargo, la medida de dispersión es bastante elevada, lo que indica valores extremos. De todas formas, esta modalidad tampoco recoge demasiadas empresas de tamaño mediano, pues el valor del percentil 75 es $9.687 .107 €$, el 75\% de empresas siguen siendo de dimensión pequeña.

Con respecto a las cooperativas y personas físicas en 2013, solo se recoge la existencia de un Centro para cada modalidad y se deben encuadrar en microempresas. Por término medio las fundaciones son pequeñas empresas, pero teniendo en cuenta que el percentil 75 es 11.036.225€, el $25 \%$ de las fundaciones se pueden encuadrar en medianas ${ }^{10}$. Las sociedades limitadas son todas de pequeña dimensión. El 25\% son empresas pequeñas, pero el resto son microempresas.

Con respecto a la componente ingreso neto de la cifra de negocios (Anexo 3), los Centros que adoptan la forma de sociedad anónima son los que obtienen mayor volumen de ventas, alcanzando una media para el año 2013 de 14.855.583€. Sin embargo, al comparar los distintos estadísticos, de nuevo se observa una elevada dispersión, el 25\% de las sociedades anónimas facturan menos de $112.000 €$ y solo el 25\% facturan más de 4.157.000€.

\footnotetext{
${ }^{10} \mathrm{Al}$ realizar el estudio de la muestra, aparecen fundaciones de gran tamaño, pero no se han tenido en cuenta por realizar actividades en diferentes provincias y Comunidades, y presentar unas únicas cuentas anuales consolidadas (Centros Especiales de Empleo creados al amparo de la Fundación ONCE).
} 
Es interesante prestar atención a la facturación del colectivo de las fundaciones: la media está en 6.066.000€, pero el percentil 75 indica cómo el $25 \%$ de este tipo de empresas supera los 16.000.000€; es decir, son empresas medianas.

Las sociedades limitadas, asociaciones, cooperativas y personas físicas, no superan la cifra de negocios de $2.000 .000 €$ al año, son todas microempresas.

\section{DESARROLLO Y CRECIMIENTO DE LOS CENTROS ESPECIALES DE EMPLEO}

Una vez que se ha realizado el estudio de la distribución y del tamaño de los CEdE atendiendo a su personalidad jurídica, se analiza el desarrollo y el crecimiento que han seguido estas entidades, principalmente a partir de 2007 (año marcado por el inicio de la crisis económica), fijando la atención en la evolución de las siguientes magnitudes económicas: activo total, cifra de negocios y resultados. Para ello se presenta el análisis de la estructura patrimonial y se recurre al balance agregado de los CEdE (Anexo 1).

En cuanto al activo agregado, se aprecia una tendencia de crecimiento a lo largo de los años 2007-2013 (Cuadro 3). Como excepción, hay que señalar que en 2013 se recoge una disminución de los activos en asociaciones y personas físicas, la cual viene originada por la desaparición de las mismas.

Cuadro 3. Evolución del activo agregado por personalidad jurídica para los CEdE de Castilla y León

\begin{tabular}{|c||c|c|c|c|c|c|c||}
\cline { 2 - 8 } \multicolumn{1}{c|}{} & $\mathbf{2 0 0 7}$ & $\mathbf{2 0 0 8}$ & $\mathbf{2 0 0 9}$ & $\mathbf{2 0 1 0}$ & $\mathbf{2 0 1 1}$ & $\mathbf{2 0 1 2}$ & $\mathbf{2 0 1 3}$ \\
\hline \hline Asociación & 26.936 .890 & 31.178 .170 & 32.608 .428 & 37.558 .250 & 39.875 .119 & 43.926 .991 & 24.679 .810 \\
\hline Cooperativa & & & & 637.326 & 1.665 .498 & 1.203 .277 & 950.723 \\
\hline Fundación & 5.796 .497 & 9.431 .046 & 8.563 .700 & 10.125 .414 & 11.356 .529 & 11.249 .081 & 13.411 .801 \\
\hline Persona Física & 257.043 & 244.045 & 205.250 & 300.389 & 316.600 & 104.507 & 132.236 \\
\hline Sociedad Anónima & 60.612 .096 & 66.709 .612 & 69.375 .168 & 172.707 .237 & 194.877 .447 & 221.359 .707 & 287.322 .703 \\
\hline Sociedad Limitada & 25.452 .005 & 35.510 .655 & 37.316 .331 & 50.581 .062 & 56.631 .882 & 69.609 .396 & 86.318 .603 \\
\hline \hline Total General & $\mathbf{1 1 9 . 0 5 4 . 5 3 2}$ & $\mathbf{1 4 3 . 0 7 3 . 5 2 7}$ & $\mathbf{1 4 8 . 0 6 8 . 8 7 6}$ & $\mathbf{2 7 1 . 9 0 9 . 6 7 8}$ & $\mathbf{3 0 4 . 7 2 3 . 0 7 4}$ & $\mathbf{3 4 7 . 4 5 2 . 9 5 8}$ & $\mathbf{4 1 2 . 8 1 5 . 8 7 7}$ \\
\hline \hline
\end{tabular}

Fuente: Elaboración propia a partir de la documentación presentada por los Centros en la Dirección de Economía Social.

Se realiza el desagregado de los componentes del activo, diferenciando entre activo no corriente y corriente para establecer una relación con la cifra de ventas. 
En el cuadro siguiente se observa como las fundaciones y los Centros cuyos titulares son personas físicas son los que muestran un activo corriente superior al activo no corriente, dato que interesa relacionar con el ingreso neto de la cifra de negocios.

Cuadro 4. Evolución del activo no corriente-activo corriente e ingreso neto de la cifra de negocios por personalidad jurídica

\begin{tabular}{|c|c|c|c|c|c|c|c|c|}
\hline & & 2007 & 2008 & 2009 & 2010 & 2011 & 2012 & 2013 \\
\hline \multirow{3}{*}{ Asociación } & ACT NC & 18.695 .846 & 20.938 .336 & 21.904 .258 & 24.984 .209 & 26.490 .137 & 28.582 .446 & 13.663 .197 \\
\hline & ACT C & 8.241 .044 & 10.239 .834 & 10.704 .170 & 12.574 .041 & 13.384 .981 & 15.344 .544 & 11.016 .613 \\
\hline & INCN & 6.416 .645 & 7.218 .140 & 8.177 .071 & 10.981 .172 & 9.680 .285 & 8.943 .383 & 9.795 .866 \\
\hline \multirow{3}{*}{ Cooperativa } & ACT NC & & & & 285.014 & 1.031 .081 & 1.029 .452 & 1.068 .399 \\
\hline & ACT C & & & & 352.313 & 634.417 & 173.825 & -117.676 \\
\hline & INCN & & & & 75.441 & 87.168 & 216.638 & 151.042 \\
\hline \multirow{3}{*}{ Fundación } & ACT NC & 1.545 .318 & 2.993 .146 & 2.665 .734 & 2.603 .225 & 3.186 .919 & 3.450 .562 & 6.073 .857 \\
\hline & ACT C & 4.251 .179 & 6.437 .900 & 5.897 .966 & 7.522 .188 & 8.169 .610 & 7.798 .519 & 7.337 .944 \\
\hline & INCN & 9.185 .326 & 10.740 .935 & 16.021 .638 & 17.997 .612 & 17.236 .947 & 13.003 .852 & 18.198 .416 \\
\hline \multirow{3}{*}{ Persona Física } & ACT NC & 107.833 & 94.416 & 72.500 & 133.269 & 72.221 & - & - \\
\hline & ACT C & 149.210 & 149.629 & 132.750 & 167.120 & 244.378 & 104.507 & 132.236 \\
\hline & INCN & 377.781 & 403.464 & 337.587 & 553.655 & 404.167 & 62.696 & 66.089 \\
\hline \multirow{3}{*}{ Sociedad Anónima } & ACT NC & 52.011 .264 & 51.978 .292 & 55.631 .655 & 127.177 .278 & 141.000 .027 & 162.073 .806 & 211.429 .310 \\
\hline & ACT C & 8.600 .833 & 14.731 .320 & 13.743 .513 & 45.529 .959 & 53.877 .420 & 59.285 .901 & 75.893 .393 \\
\hline & INCN & 45.305 .366 & 59.195 .784 & 64.760 .380 & 98.755 .269 & 130.782 .721 & 130.539 .585 & 193.122 .573 \\
\hline \multirow{3}{*}{ Sociedad Limitada } & ACT NC & 13.218 .052 & 20.125 .454 & 21.468 .338 & 29.762 .274 & 34.211 .878 & 37.847 .820 & 52.244 .288 \\
\hline & ACT C & 12.233 .954 & 15.385 .201 & 15.847 .994 & 20.818 .788 & 22.420 .005 & 31.761 .576 & 34.074 .316 \\
\hline & INCN & 25.148 .701 & 31.055 .997 & 35.130 .595 & 61.486 .423 & 74.050 .496 & 64.635 .404 & 78.420 .136 \\
\hline \multicolumn{2}{|c|}{ Total ACTIVO NO CORRIENTE } & 85.578 .313 & 96.129 .644 & 101.742 .484 & 184.945 .270 & 205.992 .262 & 232.984 .087 & 284.479 .051 \\
\hline \multicolumn{2}{|c|}{ Total ACTIVO CORRIENTE } & 33.476 .219 & 46.943 .883 & 46.326 .392 & 86.964 .408 & 98.730 .811 & 114.468 .872 & 128.336 .825 \\
\hline \multicolumn{2}{|c|}{ Total INCN } & 86.433 .819 & 108.614 .319 & 124.427 .270 & 189.849 .572 & 232.241 .783 & 217.401 .557 & 299.754 .122 \\
\hline
\end{tabular}

Fuente: Elaboración propia a partir de la documentación presentada por los Centros en la Dirección de Economía Social.

Nota. ACT C = Activo corriente; ACT NC = Activo no corriente; INCN = Ingreso neto de la cifra de negocios.

En valores absolutos (Cuadro 4) todos los Centros han incrementado la cifra de ventas durante el periodo de estudio. En 2012 ven reducida su cifra de ventas a excepción de las sociedades anónimas que se mantienen y las sociedades limitadas que siguen incrementado sus ventas de forma progresiva hasta 2013. En este mismo año se aprecia una disminución importante en la figura de persona física. Sin embargo, esta disminución no se recoge en el volumen de activo, que se incrementa en un $26,5 \%$, siendo en su totalidad activo corriente. 
En cuanto a la rotación de los activos (Cuadro 5), el estudio muestra una disminución de eficiencia en la utilización de los activos.

Cuadro 5. Evolución de la rotación de activos $\frac{I N C N}{\text { Activo total }}$, por personalidad jurídica para los CEdE de Castilla y León

\begin{tabular}{||c|c|c|c|c|c|c|c|c||}
\cline { 3 - 10 } \multicolumn{2}{c|}{} & $\mathbf{2 0 0 7}$ & $\mathbf{2 0 0 8}$ & $\mathbf{2 0 0 9}$ & $\mathbf{2 0 1 0}$ & $\mathbf{2 0 1 1}$ & $\mathbf{2 0 1 2}$ & $\mathbf{2 0 1 3}$ \\
\hline \hline Asociación & $\begin{array}{c}\text { INCN/ } \\
\text { AT }\end{array}$ & 0,24 & 0,23 & 0,25 & 0,29 & 0,24 & 0,20 & 0,40 \\
\hline Cooperativa & $\begin{array}{c}\text { INCN/ } \\
\text { AT }\end{array}$ & & & & 0,12 & 0,05 & 0,18 & 0,16 \\
\hline Fundación & $\begin{array}{c}\text { INCN/ } \\
\text { AT }\end{array}$ & 1,58 & 1,14 & 1,87 & 1,78 & 1,52 & 1,16 & 1,36 \\
\hline $\begin{array}{c}\text { Persona } \\
\text { Física }\end{array}$ & $\begin{array}{c}\text { INCN/ } \\
\text { AT }\end{array}$ & 1,47 & 1,65 & 1,64 & 1,84 & 1,28 & 0,60 & 0,50 \\
\hline $\begin{array}{c}\text { Sociedad } \\
\text { Anónima }\end{array}$ & $\begin{array}{c}\text { INCN/ } \\
\text { AT }\end{array}$ & 0,75 & 0,89 & 0,93 & 0,57 & 0,67 & 0,59 & 0,67 \\
\hline $\begin{array}{c}\text { Sociedad } \\
\text { Limitada }\end{array}$ & $\begin{array}{c}\text { INCN/ } \\
\text { AT }\end{array}$ & 0,99 & 0,87 & 0,94 & 1,22 & 1,31 & 0,93 & 0,91 \\
\hline
\end{tabular}

Fuente: Elaboración propia a partir de las cuentas anuales que presentan los CEdE.

Nota . INCN = Ingreso neto de la cifra de negocios; AT = Activo total, INCN/AT = Rotación de activos.

Los Centros que adoptan la figura de fundaciones son los que presentan un mayor índice de rotación de activos, sin embargo no son los que presentan mayores ventas (éstas se detectan en las sociedades anónimas y limitadas). Es decir, el crecimiento del índice de rotación sobre activos no viene marcado por políticas al alza de precios o por aumentos en el volumen de ventas, sino por decisiones relacionadas con el activo total de la empresa.

Cuando se observa el valor de las componentes del activo en el Cuadro 4, se comprueba la relación que existe entre activo no corriente e índice de rotación. Las fundaciones son las que presentan mayor proporción de activo corriente con respecto al no corriente, y los empresarios individuales son los que tienen mayor rotación de activos con respecto al volumen de ventas. En contraste se encuentran las asociaciones cuyo índice de rotación está en torno a un 0,25 durante todo el periodo, pero en el año 2013 se recoge un aumento en este índice de eficiencia con un valor de 0,40 .

Hay que destacar que las personas físicas son las que recogen mayor margen sobre ventas hasta 2010 (Cuadro 6). A partir de este año se da una reducción en la cifra de negocios de forma sucesiva, presentando en los últimos años un margen sobre ventas negativo, originando una reducción en la rentabilidad económica, llegando a recoger también valores negativos. 
Cuadro 6. Evolución del margen sobre ventas $\frac{B A I I}{I N C N}$, por personalidad jurídica para los CEdE de Castilla y León

\begin{tabular}{|c|c|c|c|c|c|c|c||}
\cline { 2 - 8 } \multicolumn{1}{c|}{} & $\mathbf{2 0 0 7}$ & $\mathbf{2 0 0 8}$ & $\mathbf{2 0 0 9}$ & $\mathbf{2 0 1 0}$ & $\mathbf{2 0 1 1}$ & $\mathbf{2 0 1 2}$ & $\mathbf{2 0 1 3}$ \\
\hline \hline Asociación & 0,23 & 0,12 & 0,15 & 0,04 & 0,07 & 0,00 & 0,07 \\
\hline Cooperativa & & & & $-0,06$ & $-0,75$ & 0,03 & 0,22 \\
\hline Fundación & 0,03 & 0,04 & 0,04 & 0,05 & 0,05 & 0,03 & 0,02 \\
\hline Persona Física & 0,05 & 0,14 & 0,11 & 0,19 & 0,08 & $-0,33$ & $-0,27$ \\
\hline Sociedad Anónima & 0,05 & 0,05 & 0,04 & 0,07 & 0,05 & 0,05 & 0,04 \\
\hline Sociedad Limitada & 0,03 & 0,02 & 0,03 & 0,03 & 0,03 & 0,04 & 0,08 \\
\hline
\end{tabular}

Fuente: Elaboración propia a partir de las cuentas anuales que presentan los CEdE.

En relación con el beneficio bruto, los valores obtenidos reflejan que los Centros con beneficios representan a la amplia mayoría de las empresas estudiadas apreciándose un incremento en los resultados para las sociedades anónimas y sociedades limitadas en los últimos años del estudio. El Anexo 4 recoge los estadísticos descriptivos de esta magnitud.

Las personas físicas y el 25\% de las fundaciones y sociedades limitadas presentaban resultados negativos durante todo el periodo de estudio. Las sociedades con beneficios superan el $75 \%$ del total.

\section{EMPLEO Y SUBVENCIONES EN LOS CENTROS ESPECIALES DE EMPLEO}

Es interesante el recoger un breve análisis de las magnitudes empleo y subvenciones en estas entidades, ya que una partida de sus ingresos es debida a las ayudas y subvenciones públicas concedidas, las cuales se han visto reducidas al recortar el gasto social en los presupuestos de las diferentes administraciones.

Por lo que respecta al análisis del personal contratado (Cuadro 7), a partir de 2007 se observa una tendencia general de crecimiento de empleo en todos los Centros con independencia de la forma societaria que hayan adoptado.

Las sociedades limitadas son las que registran más contratados durante todo el periodo. En el primer año de estudio presentan un 56\% del total del empleo y en 2013 alcanzan la cifra de un 64\%. Las asociaciones y las personas físicas son las que sienten más los efectos de la crisis en cuanto a la desaparición de puestos de trabajo, las asociaciones ven 
reducido el empleo en un $21,6 \%$ y los empresarios individuales en un $82 \%$. Se podría incluso pensar que este tipo de Centros tienden a desaparecer.

Cuadro 7. Evolución del empleo-gasto de personal-subvenciones por personalidad jurídica para los CEdE de Castilla y León

\begin{tabular}{|c|c|c|c|c|c|c|c|c|}
\hline & & & & & & & \\
\hline & & 2007 & 2008 & 2009 & 2010 & 2011 & 2012 & 2013 \\
\hline \multirow{4}{*}{ A } & SUBVENCIÓN & 4.338 .316 & 4.734 .665 & 5.029 .779 & 3.768 .044 & 4.303 .328 & 5.586 .683 & 6.338 .772 \\
\hline & GASTO PNAL & 6.501 .495 & 7.321 .506 & 7.865 .561 & 8.904 .431 & 9.326 .345 & 9.997 .415 & 9.511 .130 \\
\hline & $\begin{array}{l}\text { SUBVENCIÓN/ } \\
\text { GASTO PNAL }\end{array}$ & 0,67 & 0,65 & 0,64 & 0,42 & 0,46 & 0,56 & 0,67 \\
\hline & EMPLEADOS & 722 & 731 & 729 & 722 & 707 & 709 & 566 \\
\hline \multirow{4}{*}{ Coop. } & SUBVENCIÓN & & & & & & 313.058 & 390.685 \\
\hline & GASTO PNAL & & & & 5.977 & 256.266 & 467.655 & 564.363 \\
\hline & $\begin{array}{l}\text { SUBVENCIÓN/ } \\
\text { GASTO. PNAL }\end{array}$ & & & & 0,00 & 0,00 & 0,67 & 0,69 \\
\hline & EMPLEADOS & & & & 17 & 17 & 17 & 17 \\
\hline \multirow{4}{*}{$\mathrm{F}$} & SUBVENCIÓN & 122.329 & 532.069 & 573.692 & 427.741 & 664.598 & 491.331 & 976.682 \\
\hline & GASTO PNAL & 485.734 & 1.024 .647 & 1.075 .807 & 1.261 .321 & 1.650 .293 & 1.843 .778 & 2.110 .196 \\
\hline & $\begin{array}{l}\text { SUBVENCIÓN/ } \\
\text { GASTO. PNAL }\end{array}$ & 0,25 & 0,52 & 0,53 & 0,34 & 0,40 & 0,27 & 0,46 \\
\hline & EMPLEADOS & 40 & 74 & 78 & 93 & 83 & 83 & 83 \\
\hline \multirow{4}{*}{$\mathrm{PF}$} & SUBVENCIÓN & 61.350 & 62.443 & 52.693 & 76.638 & 82.978 & 24.295 & 49.574 \\
\hline & GASTO PNAL & 327.279 & 309.323 & 266.506 & 410.384 & 344.549 & 60.116 & 87.814 \\
\hline & $\begin{array}{l}\text { SUBVENCION/ } \\
\text { GASTO PNAL }\end{array}$ & 0,19 & 0,20 & 0,20 & 0,19 & 0,24 & 0,40 & 0,56 \\
\hline & EMPLEADOS & 17 & 19 & 18 & 17 & 17 & 3 & 3 \\
\hline \multirow{4}{*}{ SA } & SUBVENCIÓN & 1.924 .650 & 2.074 .335 & 2.618 .415 & 4.789 .946 & 3.105 .838 & 3.384 .912 & 5.738 .075 \\
\hline & GASTO PNAL & 5.737 .836 & 6.601 .377 & 7.544 .631 & 9.995 .914 & 10.371 .254 & 12.293 .500 & 18.047 .832 \\
\hline & $\begin{array}{l}\text { SUBVENCION/ } \\
\text { GASTO PNAL }\end{array}$ & 0,34 & 0,31 & 0,35 & 0,48 & 0,30 & 0,28 & 0,32 \\
\hline & EMPLEADOS & 335 & 356 & 423 & 474 & 471 & 466 & 412 \\
\hline \multirow{4}{*}{ SL } & SUBVENCIÓN & 6.236 .832 & 6.560 .810 & 7.401 .791 & 9.301 .237 & 12.332 .339 & 14.308 .982 & 21.693 .515 \\
\hline & GASTO PNAL & 15.555 .899 & 19.262 .282 & 22.474 .404 & 26.646 .594 & 31.795 .520 & 33.972 .104 & 41.302 .455 \\
\hline & $\begin{array}{l}\text { SUBVENCION/ } \\
\text { GASTO PNAL }\end{array}$ & 0,40 & 0,34 & 0,33 & 0,35 & 0,39 & 0,42 & 0,53 \\
\hline & EMPLEADOS & 1.399 & 1.665 & 1.889 & 1.958 & 2.000 & 1.966 & 1.905 \\
\hline \multicolumn{2}{|c|}{ TOTAL SUBVENCIÓN } & 12.683 .477 & 13.964 .323 & 15.676 .371 & 18.363 .606 & 20.489 .082 & 24.109.260 & 35.187 .302 \\
\hline \multicolumn{2}{|c|}{$\begin{array}{c}\text { TOTAL GASTO } \\
\text { PERSONAL } \\
\end{array}$} & 28.608 .242 & 34.519 .135 & 39.226 .910 & 47.224 .621 & 53.744 .227 & 58.634 .569 & 71.623 .788 \\
\hline \multicolumn{2}{|c|}{ TOTAL EMPLEADOS } & 2.513 & 2.845 & 3.137 & 3.281 & 3.294 & 3.243 & 2.986 \\
\hline
\end{tabular}

Fuente: Elaboración propia a partir de la cuenta de pérdidas y ganancias y de las memorias presentadas por los CEdE.

Nota $. \mathrm{A}=$ Asociación, $\mathrm{COOP}=$ Cooperativa, $\mathrm{F}=$ Fundación, $\mathrm{PF}=$ Persona física, $\mathrm{SA}=$ Sociedad anónima, $\mathrm{SL}=$ Sociedad limitada, PNAL= Personal 
En cuanto a las subvenciones, a pesar de los recortes en el presupuesto en relación con el gasto social público, no se observa una disminución en cuantía a lo largo del periodo estudiado. No existe una relación directa entre el número de trabajadores contratados y estas ayudas. Las subvenciones presentan un crecimiento continuo, llegando incluso las asociaciones y las cooperativas a recibir un $67 \%$ y $69 \%$, respectivamente, con respecto al gasto de personal total. Son estos Centros los que reciben un mayor importe de subvenciones en relación al gasto de personal. Las sociedades anónimas solo cubren con las subvenciones un $32 \%$ del gasto de personal para 2013.

No obstante, si se presta atención a la totalidad de las subvenciones recibidas en este año, las sociedades limitadas son las que reciben el mayor importe del total de las concedidas a estos Centros: reciben el 61,65\%, un importe bastante elevado si se compara con el resto de los CEdE. Los Centros que menos subvenciones reciben son las cooperativas, en 2013 solo percibieron un $1,1 \%$.

\section{LA CUENTA DE REVERSIÓN EN LOS CENTROS ESPECIALES DE EMPLEO}

Uno de los estudios más novedosos relacionados con los CEdE, es el realizado por Iribar y Larrinaga (2009), basado en el Informe de Impacto Social en Vizcaya elaborado por Lantegi Batuak ${ }^{11}$ en 2006, en él se lleva a cabo un análisis del impacto económico y social de la Fundación Lantegi Batuak y su CEdE en la que las personas con discapacidad son la razón y la clave de su funcionamiento.

Importante aportación de este informe es la utilización de la cuenta de reversión como una buena herramienta para medir la eficacia y el rendimiento social. Éste indicador mide que «porcentaje de todas las aportaciones que reciben los Centros de la Administración Pública es devuelto por otras vías a la sociedad» (Iribar y Larrinaga, 2009: 22).

Por tanto el desembolso real, medido en términos monetarios, que la Administración Pública realiza ayudando a los CEdE para favorecer la integración social y laboral de las PCD es la diferencia entre ingresos por subvenciones y pagos por impuestos y seguros sociales; su evolución para el periodo de estudio se muestra en el cuadro 8. La cuenta de reversión

\footnotetext{
11 Lantegi Batuak es una organización no lucrativa (ONL) que tiene como fin promover y alcanzar la integración social y laboral de las personas con discapacidad. Su origen se remonta a 1964, cuando surgen las primeras experiencias de ocupación y trabajo protegido al amparo de Gorabide, la asociación vizcaína en favor de las personas con discapacidad intelectual. La inserción laboral recoge áreas como la orientación y la formación, los centros ocupacionales y especiales de empleo, o el empleo ordinario.
} 
presenta un saldo favorable, principalmente en las sociedades mercantiles, llegando a alcanzar porcentajes superiores al $100 \%$.

Cuadro 8. Evolución de subvenciones y pagos por personalidad jurídica entre la administración y los CEdE de Castilla y León

\begin{tabular}{|c|c|c|c|c|c|c|c|c|}
\hline & & 2007 & 2008 & 2009 & 2010 & 2011 & 2012 & 2013 \\
\hline \multirow{4}{*}{ A } & SUBVENCION & 3.980 .989 & 4.335 .119 & 4.650 .156 & 3.587 .188 & 3.872 .742 & 4.558 .630 & 4.276 .218 \\
\hline & IMPUESTO & $-108,72$ & 2760,73 & 1036,31 & 3238,3 & 413,46 & $-792,45$ & 1722,47 \\
\hline & $\begin{array}{l}\text { SEGURIDAD } \\
\text { SOCIAL }\end{array}$ & 2.385 .304 & 2.662 .009 & 2.851 .149 & 3.111 .837 & 3.166 .916 & 3.287 .820 & 2.602 .819 \\
\hline & \% REVERSIÓN & $59,91 \%$ & $61,47 \%$ & $61,34 \%$ & $86,84 \%$ & $81,79 \%$ & $72,11 \%$ & $60,91 \%$ \\
\hline \multirow{4}{*}{ Coop. } & SUBVENCION & 4.318 .091 & 4.611 .042 & 4.939 .296 & 3.830 .948 & 4.074 .972 & 4.943 .307 & 4.724 .107 \\
\hline & IMPUESTO & 554 & 0 & 23058,05 & 13187,63 & 3,75 & $-21877,53$ & 11106,41 \\
\hline & $\begin{array}{l}\text { SEGURIDAD } \\
\text { SOCIAL }\end{array}$ & 123.754 & 418.852 & 428.394 & 465.930 & 538.246 & 625.792 & 525.323 \\
\hline & \% REVERSIÓN & $2,88 \%$ & $\mathbf{9 , 0 8 \%}$ & $9,14 \%$ & $12,51 \%$ & $13,21 \%$ & $12,22 \%$ & $11,36 \%$ \\
\hline \multirow{4}{*}{$\mathrm{F}$} & SUBVENCION & 4.537 .264 & 24.569 .107 & 6.347 .391 & 4.201 .697 & 5.731 .628 & 5.363 .023 & 6.534 .667 \\
\hline & IMPUESTO & 0 & 1607547 & 77711 & 208814,54 & 173827 & 85120,74 & 50394,14 \\
\hline & $\begin{array}{l}\text { SEGURIDAD } \\
\text { SOCIAL }\end{array}$ & 243.574 & 13.867 .620 & 765.770 & 845.878 & 959.846 & 1.029 .734 & 1.063 .110 \\
\hline & \% REVERSIÓN & $5,37 \%$ & $62,99 \%$ & $13,29 \%$ & $25,10 \%$ & $19,78 \%$ & $20,79 \%$ & $17,04 \%$ \\
\hline \multirow{4}{*}{$\mathrm{PF}$} & SUBVENCION & 4.581 .283 & 24.616 .390 & 6.395 .818 & 4.254 .467 & 5.782 .623 & 5.380 .919 & 6.561 .344 \\
\hline & IMPUESTO & 0 & 0 & 0 & 0 & 0 & 0 & 0 \\
\hline & $\begin{array}{l}\text { SEGURIDAD } \\
\text { SOCIAL }\end{array}$ & 91.612 & 91.690 & 97.200 & 107.571 & 73.398 & 17.713 & 18.902 \\
\hline & \% REVERSIÓN & $2,00 \%$ & $0,37 \%$ & $1,52 \%$ & $2,53 \%$ & $1,27 \%$ & $\mathbf{0 , 3 3 \%}$ & $0,29 \%$ \\
\hline \multirow{4}{*}{ SA } & SUBVENCION & 12.264 .474 & 33.267 .702 & 15.123 .626 & 18.826 .043 & 16.055 .321 & 13.653 .679 & 21.359 .868 \\
\hline & IMPUESTO & 426837 & $-43248,28$ & 923865,94 & 1149905,16 & 551802,07 & 1470621,37 & 1273877,57 \\
\hline & $\begin{array}{l}\text { SEGURIDAD } \\
\text { SOCIAL }\end{array}$ & 11.814 .041 & 14.929 .425 & 16.541 .063 & 18.169 .340 & 16.428 .612 & 16.598 .820 & 17.286 .331 \\
\hline & \% REVERSIÓN & $99,81 \%$ & $44,75 \%$ & $115,48 \%$ & $102,62 \%$ & $105,76 \%$ & $132,34 \%$ & $86,89 \%$ \\
\hline \multirow{4}{*}{ SL } & SUBVENCION & 17737049,27 & 39456470,24 & 22376417,74 & 27668225,26 & 27526359,4 & 26053574,2 & 37703400,09 \\
\hline & IMPUESTO & 241887,63 & 247755,82 & 206038,22 & 161730,71 & 490502,39 & 457705,41 & 1068295,74 \\
\hline & $\begin{array}{l}\text { SEGURIDAD } \\
\text { SOCIAL }\end{array}$ & 5.847 .758 & 7.674.226 & 9.042 .799 & 10.268 .925 & 11.919 .751 & 12.915 .133 & 13.218 .750 \\
\hline & \% REVERSIÓN & $34,33 \%$ & $20,08 \%$ & $41,33 \%$ & $37,70 \%$ & $45,08 \%$ & $51,33 \%$ & $37,89 \%$ \\
\hline \multirow{4}{*}{ TOTAL } & SUBVENCION & 47.419 .150 & 130.855 .830 & 59.832 .703 & 62.368 .568 & 63.043 .645 & 59.953 .131 & 81.159 .605 \\
\hline & IMPUESTO & 669.170 & 1.814 .815 & 1.231 .710 & 1.536 .876 & 1.216 .549 & 1.990 .778 & 2.405 .396 \\
\hline & $\begin{array}{l}\text { SEGURIDAD } \\
\text { SOCIAL }\end{array}$ & 20.506 .043 & 39.643 .822 & 29.726 .375 & 32.969 .481 & 33.086 .769 & 34.475 .012 & 34.715 .235 \\
\hline & \% REVERSIÓN & $44,66 \%$ & $31,68 \%$ & $51,74 \%$ & $55,33 \%$ & $54,41 \%$ & $60,82 \%$ & $45,74 \%$ \\
\hline
\end{tabular}

Fuente: Elaboración propia a partir de la cuenta de pérdidas y ganancias y de las memorias presentadas por los CEdE.

Nota. $A=$ Asociación, $C O O P=$ Cooperativa,$F=$ Fundación, $P F=$ Persona física, $S A=$ Sociedad anónima, $S L$ $=$ Sociedad limitada.

\section{CONCLUSIONES}

Los Centros Especiales de Empleo son empresas que tienen como principal objetivo el realizar una actividad productiva, participando de forma regular en las operaciones de mercado, y donde su estructura y organización pueden ser idénticas que las de las empresas 
ordinarias, de ahí la importancia de realizar un estudio económico-financiero. Además tienen como finalidad el asegurar un empleo remunerado para las personas con discapacidad; a la vez que son un medio de inclusión del mayor número de estas personas en el régimen de empleo ordinario.

Por tanto los Centros Especiales de Empleo son empresas recogidas en el sector de la Economía Social por su compromiso social hacia colectivos con menores oportunidades en el mercado de trabajo.

A la vista de los datos obtenidos en el desarrollo del trabajo, se aprecia de forma clara que la sociedad limitada es la forma jurídica con mayor presencia con respecto al total de los CEdE de Castilla y León (74\% en 2013). A lo largo de los periodos analizados se observa que la proporción en cuanto al tipo societario no ha variado sustancialmente, pero que a partir de 2008 empiezan a desaparecer los empresarios individuales y surgen otras formas como cooperativas y asociaciones con actividad empresarial.

En cuanto al crecimiento y desarrollo de estas organizaciones, se aprecia que las sociedades anónimas y limitadas son los Centros que presentan valores más favorables durante todos los años analizados. Estas entidades no se ven afectadas por la crisis y continua su crecimiento en activos, cifra de ventas y beneficios de una manera progresiva desde 2007. Por el contrario, son las asociaciones y las personas físicas las entidades que presentan una importante reducción en las magnitudes económicas mencionadas.

El objetivo fundamental de toda política de crecimiento debería ir orientada a conseguir que la cifra de ventas crezca más que las inversiones en activo, puesto que una acción dirigida a un incremento de ventas, si va acompañada de un elevado aumento en los activos no corrientes, supone inevitablemente una reducción de la rentabilidad económica al incrementarse el denominador del índice de rotación de los activos. Esto sucede en los CEdE, a partir de 2010 se reduce la rentabilidad económica y solamente las sociedades limitadas presentan una rentabilidad aceptable, la cual se debe a un incremento del margen sobre ventas, ya que la rotación de activos soporta una considerable reducción.

El estudio muestra una disminución de eficiencia en la utilización de los activos de los Centros, es decir, el crecimiento del índice de rotación sobre activos no viene marcado por políticas al alza de precios o por aumentos en el volumen de ventas, sino por decisiones relacionadas con el activo total de la empresa. 
Al estudiar la cifra de resultados, hay que destacar que las fundaciones son los Centros más afectados por la crisis de 2007. Esta situación viene originada además por la propia naturaleza de estas entidades, ya que en situaciones complicadas éstas tienen que hacer frente al aumento de los beneficiarios y de nuevas necesidades sociales, además de disponer de menores recursos.

En relación a la magnitud empleo, los efectos de la crisis empiezan a sentirse en 2010, año en que se registra la mayor cifra de empleo de personas con discapacidad, descendiendo el número de trabajadores en todos los Centros de la comunidad hasta en un $9 \%$ en valor absoluto. No obstante a pesar de esta importante reducción, los Centros que presentan la forma de sociedad limitada son los que alcanzan la máxima contratación de trabajadores con discapacidad.

En cuanto a las subvenciones, a pesar de los recortes en el presupuesto en relación con el gasto social público, no se observa una disminución en cuantía a lo largo del periodo estudiado. Del estudio se deduce que no se puede establecer una relación causa-efecto entre la reducción de subvenciones y el número de trabajadores: las subvenciones no solo no se reducen, sino que se incrementan de forma importante, exceptuando el caso de los empresarios individuales, en los que la cuantía de las subvenciones recibidas se ve reducida a la vez que el número de trabajadores empleados.

Se concluye estableciendo que la crisis si afecta a los CEdE, sin diferenciar la figura jurídica en la que se han constituido y que la reducción en el número de trabajadores no viene originado por una política restrictiva en la política de subvenciones, siendo las entidades más perjudicadas asociaciones y personas físicas, pudiendo incluso ocasionar su cierre.

\section{REFLEXION FINAL}

No se puede atribuir la reducción en el empleo a la reducción de las subvenciones concedidas a los CEdE en la comunidad de Castilla y León, ya que éstas siguen creciendo para el periodo de estudio 2007-2013. Lo cual podría abrir un debate interesante, ya que si el objetivo principal de estas entidades es la contratación de PCD, ¿por qué disminuye el número de empleados cuando las subvenciones concedidas no hace sino aumentar?, ¿Quizá estas subvenciones han ido dirigidas a un incremento de activos no corrientes como fomento a la actividad empresarial? 
Son muchos los beneficios sociales, laborales y económicos que los CEdE aportan a las administraciones públicas, a las PCD, a sus familias y para el conjunto de la sociedad. Al realizar una actividad, los CEdE generan riqueza y desarrollo económico como cualquier empresa ordinaria, compitiendo en el mercado por vender sus productos o servicios, contribuyendo de forma directa a través de la imposición tributaria y de las aportaciones al sistema de pensiones. Con los impuestos (el de sociedades e impuesto sobre el valor añadido) y cotizaciones, están compensando parte de las ayudas públicas que reciben. Asimismo, los CEdE generan empleo con el correspondiente ahorro público, al convertir posibles perceptores de servicios sociales en trabajadores, contribuyentes y consumidores.

Todo ello hace que las ayudas económicas e inversiones públicas deban seguir impulsando políticas activas de empleo en relación al empleo protegido de estas personas, y por ello es necesaria la colaboración por parte del sector público y privado.

\section{BIBLIOGRAFÍA}

\section{AECA. ASOCIACIÓN ESPAÑOLA DE CONTABILIDAD Y ADMINISTRACIÓN DE} EMPRESAS (1991 r) Principios y Normas de Contabilidad en España. Principios Contables, Documento núm. 1, AECA, Madrid.

AMAT SALAS, O. (2008) Análisis de estados financieros, Barcelona. Gestión $20008^{\mathrm{a}}$ Edición.

CALVO VÉRGEZ, J. (2004) Centros especiales de empleo: situación jurídica actual y perspectivas de futuro. GEZKI No 0, pp. 139-160.

CAMACHO MIÑANO, M.M., SEGOVIA VARGAS, M.J. y GELASHVILI V. (2015) Patrones de Supervivencia para la gestión de los centros especiales de empleo. Revista de Estudios Empresariales. Segunda época, № 1, pp. 109-126.

CARRIO FERNANDEZ, E.M. (2005) Análisis descriptivo de los centros especiales de empleo en el principado de Asturias. Revista de investigación educativa, Volumen $\mathrm{N}^{\mathrm{a}} 23$, pp. 41-55.

GÓMEZ GARCÍA, J. M., ROMÁN ORTEGA, A. y ROJO JIMÉNEZ, C. (2010) Las dimensiones de la calidad del empleo en la economía social: un análisis de las Sociedades Laborales y de los Centros Especiales de Empleo en Castilla y León. CIRIEC-España, Revista de Economía Pública Social y Cooperativa, № 67. 
GÓNZALEZ HEREDIA, J. (2008) Análisis de eficiencia y productividad en entidades del Tercer Sector: Los Centros Especiales de Empleo. Universidad Politécnica de Cartagena. D. de Economía Financiera y Contabilidad.

IRIBAR BILBAO, M.F. y LARRINAGA OJANGUREN, M.A. (2009) Casos de gestión avanzada. Foro Conocimiento Empresa-Universidad. Lantegi Batuak 100\% capaces. INNOBASQUE.

JORDÁN DE URRIES Y VERDUGO (2010) Informe sobre la situación de los Centros Especiales de Empleo en España. Universidad de Salamanca: Instituto Universitario de Integración de la Comunidad.

KPMG (2007-2013) Presente y futuro de los Centros Especiales de Empleo. Asesores en el marco del Programa Operativo del Fondo Social Europeo de Lucha contra la Discriminación, y cofinanciado por Fundación ONCE y el Ministerio de Empleo y Seguridad Social; con la colaboración de la Federación Empresarial Española de Asociaciones de Centros de Empleo (FEACEM).

RÍO, D. del (1984) Aproximación Socio Laboral al Empleo Protegido: Los Centros Especiales de Empleo. GPS. Madrid.

RODRÍGUEZ ENRÍQUEZ, E. (2002) Análisis económico-financiero del sector de automoción en España. Boletín económico de ICE núm. 2747, pp. 11-17.

RODRÍGUEZ ENRÍQUEZ, E. (2003) Comportamiento económico-financiero de las empresas constructoras en Andalucía: un estudio comparativo por provincias. Boletín Económico de Andalucía, pp. 417-433, 23 Ref.

RODRÍGUEZ ALVAREZ, V. (2012) El empleo de las personas con discapacidad en la gran recesión: ¿Son los Centros Especiales de Empleo una excepción?. Estudios de Economía Aplicada, no 1 (30), pp. 10-24.

RUBIO ARRIBAS, F.J. (2003) La Construcción social del empleo protegido: Los Centros Especiales de Empleo. Nómadas Revista Crítica de Ciencias Sociales y Jurídicas núm. 008, Universidad Complutense de Madrid.

SOMOZA LÓPEZ, A. y VALLVERDÚ CALAFELL, J. (2003) Una comparación de la Selección de los Ratios Contables en los modelos contables financieros de predicción de la insolvencia empresarial, Documents de treball de la divisió de ciències jurídiques e conòmiques i social. Departamento de Economía Financiera y de Contabilidad, Universidad de Barcelona. 
TUSET DEL PINO, P. (2002) El Centro Especial de Empleo, Naturaleza y Clases. Constitución y Registro. Objeto. Gestión y Financiación. Publicaciones Jover Abogados, Barcelona.

VISIER, L. (1998) Relaciones laborales en los sistemas de trabajo protegido para personas minusválidas. Revista Internacional del Trabajo, Vol. 117.

\section{LEGISLACIÓN CONSULTADA}

- Orden de 7 de noviembre de 1968, sobre Centros Especiales de Empleo para Trabajadores Minusválidos (BOE núm. 288, de 30 de noviembre 1968).

- Real Decreto 2531/1970, de 22 de agosto, desarrollada por la Orden de 12 de enero de 1972, sobre empleo de trabajadores con discapacidad (BOE núm. 221, de 15 de septiembre 1970).

- Ley 13/1982, de 7 de abril. Integración social de los minusválidos (LISMI), (BOE núm. 103, de 30 de abril 1982).

- Real Decreto 832/1995, de 30 de mayo, por el que se traspasó a la Comunidad Autónoma de Castilla y León la gestión de los programas de apoyo al empleo, entre los que se encuentra el de integración laboral de personas con discapacidad en Centros Especiales de Empleo (BOE núm. 160 de 6 de julio 1995).

- Real Decreto 1368/1985, de 17de julio, por el que se regula la relación laboral de carácter especial de los minusválidos que trabajen en los Centros Especiales de Empleo, (BOE núm. 189, de 8 de agosto 1985).

- Real Decreto 2273/1985, de 4 de diciembre, por el que se aprueba el Reglamento de los Centros Especiales de Empleo, definidos en el Art. 42 de la ley 13/1982, de 7 de abril, de integración social del minusválido, (BOE núm. 294, de 9 de diciembre 1985).

- Plan de Acción para las Personas con Discapacidad (2006-2015): Un Plan sobre cómo mejorar la vida de las personas con discapacidad en Europa. Centro de Referencia Estatal de Autonomía Personal y Ayudas Técnicas (CEAPAT) del Instituto de Mayores y Servicios Sociales (IMSERSO), Ministerio de Sanidad, Política Social e Igualdad, en colaboración con la Federación de Organizaciones en favor de las personas con discapacidad intelectual de Madrid, abril 2006.

- Orden de 3 de enero de 2001 de la Consejería de Industria, Comercio y Turismo, por la que se crea el Registro de Centros Especiales de Empleo de la Comunidad de Castilla y León. (BOCyL núm. 23, de 1 de febrero 2001).

- Ley 39/2006, de 14 de diciembre, de promoción de la Autonomía Personal y Atención a las personas en situación de dependencia, (BOE número 299, de 15 de diciembre 2006). 
- Orden EYE/500/2007, de 15 de febrero, por la que se crea y regula el fichero de datos de carácter personal derivado del Registro de Centros Especiales de Empleo (BOCYL núm. 59 de 23 de marzo 2007).

- Real Decreto 1514/2007, de 16 de noviembre, por el que se aprueba el Plan General de Contabilidad (BOE núm. 278, de 20 de noviembre de 2007).

- Real Decreto 1515/2007, de 16 de noviembre, por el que se aprueba el Plan General de Contabilidad de Pequeñas y Medianas Empresas y los criterios contables específicos para microempresas (BOE núm. 279, de 21 de noviembre de 2007).

- Orden EYE/1847/2008, de 14 de octubre, por la que se modifica la Orden EYE 500/2007, de 15 de febrero, por la que se crea y regula el fichero de datos de carácter personal derivado del Registro de Centros Especiales de Empleo (BOCYL núm. 210, de 14 de octubre 2008).

- Orden EYE/2335/2009, de 21 de diciembre, por la que se adecua el Real Decreto 469/2006, de 21 de abril, por el que se regulan las unidades de apoyo a la actividad profesional en el marco de los servicios de ajuste personal y social de los Centros Especiales de Empleo a las peculiaridades organizativas y la normativa aplicable en la Comunidad de Castilla y León (BOCYL. núm. 246, de 24 de diciembre de 2009).

- Real Decreto Legislativo 1/2013, de 29 de noviembre, por el que se aprueba el Texto Refundido de la Ley General de derechos de las personas con discapacidad y de su inclusión social (BOE núm. BOE» núm. 289, de 3 de diciembre de 2013). 
ANEXO 1: EVOLUCIÓN DE LAS DIVERSAS PARTIDAS DEL BALANCE PARA LOS CENTROS ESPECIALES DE EMPLEO POR PERSONALIDAD JURÍDICA. AÑOS: 2007-2013

1.1 ASOCIACIONES

\begin{tabular}{|c|c|c|c|c|c|c|c|}
\hline & 2007 & 2008 & 2009 & 2010 & 2011 & 2012 & 2013 \\
\hline TOTAL ACTIVO & 26.936 .890 & 31.178 .170 & 32.793 .808 & 37.558 .250 & 39.875 .119 & 43.926 .991 & 24.679 .810 \\
\hline A). ACTIVO NO CORRIENTE & 18.695 .846 & 20.938 .336 & 21.958 .192 & 24.984 .209 & 26.490 .137 & 28.582 .446 & 13.663 .197 \\
\hline INMOVILIZADO & 17.785 .531 & 19.534 .365 & 20.799 .315 & 24.412 .661 & 25.493 .018 & 28.521 .664 & 13.627 .571 \\
\hline I- Inmovilizado Intangible & 80.604 & 59.973 & 178.197 & 166.776 & 102.919 & 81.457 & 82.144 \\
\hline II- Inmovilizado material & 17.682 .587 & 19.464 .089 & 20.611 .655 & 23.607 .083 & 25.152 .048 & 27.155 .978 & 10.402 .418 \\
\hline V- Inmovilizado Financiero & 23.503 & 10.303 & 9.463 & 638.802 & 238.051 & 1.284 .229 & 3.143 .008 \\
\hline $\begin{array}{c}\text { III+IV+VI- Otros activos fijos no } \\
\text { corrientes }\end{array}$ & 910.315 & 1.403 .971 & 1.158 .878 & 571.548 & 997.119 & 60.782 & 35.627 \\
\hline B). ACTIVO CORRIENTE & 8.241 .044 & 10.239 .834 & 10.835 .616 & 12.574 .041 & 13.384 .981 & 15.344 .544 & 11.016 .613 \\
\hline TOTAL PASIVO & 26.936 .890 & 31.178 .170 & 32.793 .808 & 37.558 .250 & 39.875 .119 & 43.926 .991 & 24.679 .810 \\
\hline A) PATRIMONIO NETO & 20.552 .142 & 22.565 .046 & 24.428 .229 & 26.851 .809 & 29.080 .577 & 33.106 .537 & 19.475 .891 \\
\hline A.1.) FONDOS PROPIOS & 10.258 .386 & 10.914 .491 & 12.578 .015 & 11.821 .170 & 10.640 .105 & 15.307 .180 & 9.609 .996 \\
\hline B) PASIVO NO CORRIENTE & 2.118 .415 & 2.911 .231 & 2.841 .392 & 2.816 .883 & 2.757 .842 & 2.768 .591 & 1.685 .947 \\
\hline C) PASIVO CORRIENTE & 4.266 .333 & 5.701 .893 & 5.524 .186 & 7.889 .559 & 8.036 .700 & 8.051 .863 & 3.517 .972 \\
\hline
\end{tabular}

Fuente: Elaboración propia a partir de las cuentas anuales presentadas por los CEdE en la Dirección General de Economía Social.

1.2. COOPERATIVAS

\begin{tabular}{|c|c|c|c|c|c|c|c|}
\hline & 2007 & 2008 & 2009 & 2010 & 2011 & 2012 & 2013 \\
\hline TOTAL ACTIVO & & & & 637.326 & 1.665 .498 & 1.203 .277 & 950.723 \\
\hline $\begin{array}{l}\text { A). ACTIVO NO } \\
\text { CORRIENTE }\end{array}$ & & & & 285.014 & 1.031 .081 & 1.029 .452 & 1.068 .399 \\
\hline INMOVILIZADO & & & & 285.014 & 1.031 .081 & 1.029 .452 & 1.068 .399 \\
\hline I- Inmovilizado Intangible & & & & 0 & 0 & 0 & 0 \\
\hline II- Inmovilizado material & & & & 285.014 & 1.031 .081 & 1.029 .452 & 1.068 .399 \\
\hline V- Inmovilizado Financiero & & & & 0 & 0 & 0 & 0 \\
\hline $\begin{array}{l}\text { III+IV+VI- Otros activos fijos } \\
\text { no corrientes }\end{array}$ & & & & 0 & 0 & 0 & 0 \\
\hline B). ACTIVO CORRIENTE & & & & 352.313 & 634.417 & 173.825 & -117.676 \\
\hline TOTAL PASIVO & & & & 637.326 & 1.665 .498 & 1.203 .277 & 950.723 \\
\hline A) PATRIMONIO NETO & & & & 214.408 & 685.291 & 672.998 & 746.134 \\
\hline A.1.) FONDOS PROPIOS & & & & 1.051 & -64.015 & -54.793 & -20.148 \\
\hline B) PASIVO NO CORRIENTE & & & & 0 & 0 & 0 & 0 \\
\hline C) PASIVO CORRIENTE & & & & 422.919 & 980.207 & 530.279 & 204.589 \\
\hline
\end{tabular}


Fuente: Elaboración propia a partir de las cuentas anuales presentadas por los CEdE en la Dirección General de Economía Social.

\subsection{FUNDACIONES}

\begin{tabular}{|c|c|c|c|c|c|c|c|}
\hline & 2007 & 2008 & 2009 & 2010 & 2011 & 2012 & 2013 \\
\hline TOTAL ACTIVO & 5.796 .497 & 9.431 .046 & 8.596 .202 & 10.125 .414 & 11.356 .529 & 11.249 .081 & 13.411 .801 \\
\hline A). ACTIVO NO CORRIENTE & 1.545 .318 & 2.993 .146 & 2.690 .750 & 2.603 .225 & 3.186 .919 & 3.450 .562 & 6.073 .857 \\
\hline INMOVILIZADO & 1.545 .318 & 2.992 .949 & 2.667 .386 & 2.603 .225 & 3.418 .539 & 3.729 .480 & 4.433 .638 \\
\hline I- Inmovilizado Intangible & 12.205 & 52.219 & 42.656 & 5.852 & 19.216 & 23.267 & 71.064 \\
\hline II- Inmovilizado material & 1.533 .114 & 2.807 .223 & 2.623 .773 & 2.556 .058 & 3.363 .710 & 3.706 .212 & 4.361 .399 \\
\hline V- Inmovilizado Financiero & 0 & 133.506 & 957 & 41.315 & 35.613 & 0 & 1.175 \\
\hline $\begin{array}{c}\text { III+IV+VI- Otros activos fijos no } \\
\text { corrientes }\end{array}$ & 0 & 197 & 23.364 & 0 & -231.620 & -278.918 & 1.640 .219 \\
\hline B). ACTIVO CORRIENTE & 4.251 .179 & 6.437 .900 & 5.905 .452 & 7.522 .188 & 8.169 .610 & 7.798 .519 & 7.337 .944 \\
\hline TOTAL PASIVO & 5.796 .497 & 9.431 .046 & 8.596 .202 & 10.125 .414 & 11.356 .529 & 11.249 .081 & 13.411 .801 \\
\hline A) PATRIMONIO NETO & 621.185 & 2.363 .359 & 2.830 .024 & 3.401 .758 & 4.050 .739 & 4.702 .793 & 6.610 .991 \\
\hline A.1.) FONDOS PROPIOS & 464.215 & 2.238 .256 & 2.682 .372 & 2.907 .906 & 3.635 .529 & 4.115 .477 & 4.950 .708 \\
\hline B) PASIVO NO CORRIENTE & 2.629 .514 & 2.747 .580 & 1.099 .788 & 1.030 .472 & 2.474 .579 & 599.232 & 625.083 \\
\hline C) PASIVO CORRIENTE & 2.545 .798 & 4.320 .108 & 4.666 .390 & 5.693 .184 & 4.831 .211 & 5.947 .056 & 6.175 .728 \\
\hline
\end{tabular}

Fuente: Elaboración propia a partir de las cuentas anuales presentadas por los CEdE en la Dirección General de Economía Social.

1.4. PERSONA FÍSICA

\begin{tabular}{|c|c|c|c|c|c|c|c|}
\hline & & & & & & & \\
\hline & 2007 & 2008 & 2009 & 2010 & 2011 & 2012 & 2013 \\
\hline TOTAL ACTIVO & 244.045 & 257.043 & 205.250 & 300.389 & 316.600 & 104.507 & 132.236 \\
\hline A). ACTIVO NO CORRIENTE & 94.416 & 107.833 & 72.500 & 133.269 & 72.221 & 0 & 0 \\
\hline INMOVILIZADO & 94.416 & 107.833 & 72.500 & 133.269 & 72.221 & 0 & 0 \\
\hline I- Inmovilizado Intangible & 0 & 0 & 0 & 0 & 0 & 0 & 0 \\
\hline II- Inmovilizado material & 94.416 & 107.833 & 72.500 & 133.269 & 72.221 & 0 & 0 \\
\hline V- Inmovilizado Financiero & 0 & 0 & 0 & 0 & 0 & 0 & 0 \\
\hline $\begin{array}{c}\text { III+IV+VI- Otros activos fijos no } \\
\text { corrientes }\end{array}$ & 0 & 0 & 0 & 0 & 0 & 0 & 0 \\
\hline B). ACTIVO CORRIENTE & 149.629 & 149.210 & 132.750 & 167.120 & 244.378 & 104.507 & 132.236 \\
\hline TOTAL PASIVO & 244.045 & 257.043 & 205.250 & 300.389 & 316.600 & 104.507 & 132.236 \\
\hline A) PATRIMONIO NETO & 160.987 & 179.234 & 114.797 & 218.316 & 32.838 & -20.588 & -17.890 \\
\hline A.1.) FONDOS PROPIOS & 160.987 & 179.234 & 114.797 & 218.316 & 32.838 & -20.588 & -17.890 \\
\hline B) PASIVO NO CORRIENTE & 0 & 0 & 0 & 0 & 0 & 0 & 0 \\
\hline C) PASIVO CORRIENTE & 83.058 & 77.809 & 90.453 & 82.073 & 283.761 & 125.095 & 150.126 \\
\hline
\end{tabular}

Fuente: Elaboración propia a partir de las cuentas anuales presentadas por los CEdE en la Dirección General de Economía Social. 


\subsection{SOCIEDAD ANÓNIMA}

\begin{tabular}{|c|c|c|c|c|c|c|c|}
\hline & 2007 & 2008 & 2009 & 2010 & 2011 & 2012 & 2013 \\
\hline TOTAL ACTIVO & 60.612 .096 & 66.709 .612 & 69.401 .796 & 172.707 .237 & 194.877 .447 & 221.359 .707 & 287.322 .703 \\
\hline A). ACTIVO NO CORRIENTE & 52.011 .264 & 51.978 .292 & 55.640 .670 & 127.177.278 & 141.000 .027 & 162.073 .806 & 211.429 .310 \\
\hline INMOVILIZADO & 52.009 .205 & 51.920 .567 & 54.760 .458 & 127.123 .985 & 135.642 .734 & 161.680 .898 & 200.742 .918 \\
\hline I- Inmovilizado Intangible & 760.935 & 762.249 & 3.176 .656 & 3.837 .984 & 8.845 .997 & 9.460 .455 & 10.681 .722 \\
\hline II- Inmovilizado material & 51.201 .008 & 50.056 .824 & 46.575 .811 & 107.280 .960 & 117.201 .832 & 136.454.109 & 178.690 .491 \\
\hline V- Inmovilizado Financiero & 49.416 & 1.101 .493 & 5.007 .992 & 16.005 .042 & 9.594 .906 & 15.766 .335 & 11.370 .704 \\
\hline $\begin{array}{c}\text { III+IV+VI- Otros activos fijos no } \\
\text { corrientes }\end{array}$ & 2.059 & 57.724 & 880.212 & 53.293 & 5.357 .292 & 392.908 & 10.686 .392 \\
\hline B). ACTIVO CORRIENTE & 8.600 .833 & 14.731 .320 & 13.761 .126 & 45.529 .959 & 53.877 .420 & 59.285 .901 & 75.893 .393 \\
\hline TOTAL PASIVO & 60.612 .096 & 66.709 .612 & 69.401 .796 & 172.707 .237 & 194.877 .447 & 221.359 .707 & 287.322 .703 \\
\hline A) PATRIMONIO NETO & 28.757 .083 & 34.902 .323 & 36.653 .948 & 76.379 .907 & 91.481 .465 & 105.166.905 & 124.885 .912 \\
\hline A.1.) FONDOS PROPIOS & 15.489 .447 & 33.728 .427 & 22.318 .672 & 73.939 .057 & 71.715 .943 & 84.218 .978 & 99.077 .875 \\
\hline B) PASIVO NO CORRIENTE & 15.584 .885 & 15.771 .667 & 13.526 .225 & 59.049 .152 & 68.349 .515 & 75.409 .885 & 105.004 .694 \\
\hline C) PASIVO CORRIENTE & 16.270 .128 & 16.035 .622 & 19.221 .623 & 37.278 .179 & 35.046 .467 & 40.782 .917 & 57.432 .098 \\
\hline
\end{tabular}

Fuente: Elaboración propia a partir de las cuentas anuales presentadas por los CEdE en la Dirección General de Economía Social.

1.6. SOCIEDAD LIMITADA

\begin{tabular}{|c|c|c|c|c|c|c|c|}
\hline & 2007 & 2008 & 2009 & 2010 & 2011 & 2012 & 2013 \\
\hline TOTAL ACTIVO & 25.452 .005 & 35.510 .655 & 37.373 .999 & 50.581 .062 & 56.631 .882 & 69.609 .396 & 86.318 .603 \\
\hline A). ACTIVO NO CORRIENTE & 13.218 .052 & 20.125 .454 & 21.488 .525 & 29.762 .274 & 34.211 .878 & 37.847 .820 & 52.244 .288 \\
\hline INMOVILIZADO & 12.916 .937 & 19.256 .820 & 20.409 .073 & 28.200 .758 & 33.204 .419 & 35.988 .632 & 51.275 .470 \\
\hline I- Inmovilizado Intangible & 459.255 & 1.080 .800 & 1.463 .827 & 2.442 .681 & 693.250 & 736.376 & 1.043 .756 \\
\hline II- Inmovilizado material & 12.322 .323 & 18.015 .604 & 18.762 .374 & 24.398 .784 & 31.164 .943 & 33.881 .932 & 46.679 .473 \\
\hline V- Inmovilizado Financiero & 135.539 & 160.416 & 182.873 & 1.359 .292 & 1.346 .226 & 1.370 .324 & 3.552 .241 \\
\hline $\begin{array}{c}\text { III+IV+VI- Otros activos fijos no } \\
\text { corrientes }\end{array}$ & 301.115 & 868.634 & 1.079 .452 & 1.561 .516 & 1.007 .459 & 1.859 .188 & 968.818 \\
\hline B). ACTIVO CORRIENTE & 12.233 .954 & 15.385 .201 & 15.885 .474 & 20.818 .788 & 22.420 .005 & 31.761 .576 & 34.074 .316 \\
\hline TOTAL PASIVO & 25.452 .005 & 35.510 .654 & 37.373 .999 & 50.581 .061 & 56.631 .882 & 69.609 .396 & 86.318 .603 \\
\hline A) PATRIMONIO NETO & 8.414 .669 & 8.956 .723 & 11.682 .861 & 15.685 .128 & 17.336 .757 & 19.249 .952 & 32.586 .919 \\
\hline A.1.) FONDOS PROPIOS & 5.336 .712 & 5.004 .020 & 6.773 .810 & 10.341 .763 & 11.338 .027 & 13.313 .946 & 22.196 .981 \\
\hline B) PASIVO NO CORRIENTE & 4.349 .267 & 9.607 .144 & 10.145 .766 & 14.279 .901 & 16.841 .641 & 23.065 .017 & 24.770 .316 \\
\hline C) PASIVO CORRIENTE & 12.688 .070 & 16.946 .787 & 15.545.372 & 20.616 .032 & 22.453 .256 & 27.294.427 & 28.961 .369 \\
\hline
\end{tabular}

Fuente: Elaboración propia a partir de las cuentas anuales presentadas por los CEdE en la Dirección General de Economía Social. 
ANEXO 2. EVOLUCIÓN DE LOS ESTADÍSTICOS PARA EL ACTIVO AGREGADO DE LOS CEDE. FIGURA JURÍDICA

\begin{tabular}{|c|c|c|c|c|c|c|c|c|}
\hline & & 2007 & 2008 & 2009 & 2010 & 2011 & 2012 & 2013 \\
\hline \multirow[t]{5}{*}{ ASO } & Media & 2.244 .741 & 2.598 .181 & 2.717 .369 & 3.129 .854 & 3.322 .927 & 3.660 .583 & 2.742 .201 \\
\hline & $\begin{array}{c}\text { Desviación } \\
\text { típica }\end{array}$ & 3.416 .585 & 4.324 .370 & 4.179.267 & 4.402 .649 & 4.914 .500 & 5.389 .345 & 2.238.409 \\
\hline & Percentil 75 & 2.069 .605 & 2.010 .379 & 2.507 .992 & 3.664 .493 & 2.967 .533 & 2.868 .026 & 2.930 .562 \\
\hline & Mediana & 1.360 .780 & 1.629 .984 & 1.630 .863 & 1.719 .862 & 2.028 .753 & 2.385 .240 & 2.830 .917 \\
\hline & Percentil 25 & 193.393 & 179.039 & 213.488 & 646.449 & 819.607 & 1.026 .822 & 1.096 .345 \\
\hline \multirow{5}{*}{ COOP } & Media & & & & 637.326 & 1.665 .498 & 1.203 .277 & 950.723 \\
\hline & $\begin{array}{c}\text { Desviación } \\
\text { típica }\end{array}$ & & & & ras & 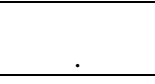 & & . \\
\hline & Percentil 75 & & & & 637.326 & 1.665 .498 & 1.203 .277 & 950.723 \\
\hline & Mediana & & & & 637.326 & 1.665 .498 & 1.203 .277 & 950.723 \\
\hline & Percentil 25 & & & & 637.326 & 1.665 .498 & 1.203 .277 & 950.723 \\
\hline \multirow[t]{5}{*}{ FUN } & Media & 2.898.249 & 3.143 .682 & 2.854 .567 & 3.375 .138 & 3.785 .510 & 3.749 .694 & 4.470 .600 \\
\hline & $\begin{array}{c}\text { Desviación } \\
\text { típica }\end{array}$ & 4.079 .408 & 3.848 .296 & 3.582 .773 & 4.576 .596 & 5.157 .204 & 4.951 .180 & 5.708 .078 \\
\hline & Percentil 75 & 5.782 .826 & 7.495.540 & 6.934 .129 & 8.625 .406 & 9.705 .893 & 9.444 .986 & 11.036 .225 \\
\hline & Mediana & 2.898.249 & 1.745 .815 & 1.409 .880 & 1.270 .811 & 1.380 .789 & 1.334 .384 & 1.689 .373 \\
\hline & Percentil 25 & 13.671 & 189.691 & 219.691 & 229.197 & 269.847 & 469.711 & 686.203 \\
\hline \multirow{5}{*}{$\begin{array}{c}\text { PERSONA } \\
\text { FISICA }\end{array}$} & Media & 128.521 & 122.022 & 102.625 & 150.194 & 158.300 & 104.507 & 132.236 \\
\hline & $\begin{array}{c}\text { Desviación } \\
\text { típica }\end{array}$ & 84.111 & 51.081 & 19.181 & 94.812 & 117.345 & & . \\
\hline & Percentil 75 & 187.997 & 158.142 & 116.188 & 217.237 & 241.275 & 104.507 & 132.236 \\
\hline & Mediana & 128.521 & 122.022 & 102.625 & 150.194 & 158.300 & 104.507 & 132.236 \\
\hline & Percentil 25 & 69.046 & 85.903 & 89.062 & 83.152 & 75.325 & 104.507 & 132.236 \\
\hline \multirow{5}{*}{ SA } & Media & 5.510 .191 & 5.131 .509 & 4.955 .369 & 10.794 .202 & 12.991 .830 & 14.757 .314 & 22.101 .746 \\
\hline & $\begin{array}{c}\text { Desviación } \\
\text { típica }\end{array}$ & 14.154 .906 & 14.017 .235 & 13.558 .370 & 25.507 .434 & 30.545 .775 & 33.840 .288 & 47.447 .256 \\
\hline & Percentil 75 & 4.067 .140 & 2.186 .433 & 3.051 .276 & 5.238 .341 & 5.684 .843 & 7.028 .879 & 9.687 .107 \\
\hline & Mediana & 891.376 & 545.672 & 620.600 & 999.076 & 869.615 & 942.391 & 1.344 .679 \\
\hline & Percentil 25 & 106.208 & 193.860 & 209.622 & 353.180 & 244.729 & 263.038 & 305.438 \\
\hline \multirow{5}{*}{ SL } & Media & 480.227 & 572.752 & 533.090 & 632.263 & 690.633 & 859.375 & 1.150 .915 \\
\hline & $\begin{array}{c}\text { Desviación } \\
\text { típica }\end{array}$ & 840.859 & 1.498 .212 & 1.339 .612 & 1.369 .098 & 1.377 .160 & 2.114 .706 & 3.057 .201 \\
\hline & Percentil 75 & 549.183 & 523.845 & 546.929 & 757.544 & 798.909 & 894.812 & 1.168 .159 \\
\hline & Mediana & 200.380 & 246.409 & 290.594 & 302.894 & 348.976 & 304.036 & 390.881 \\
\hline & Percentil 25 & 54.074 & 63.911 & 61.618 & 95.297 & 112.177 & 160.500 & 190.776 \\
\hline
\end{tabular}

Fuente: Elaboración propia a partir de los Balances presentados por los CEdE.

Nota: ASO: Asociación, COOP: Cooperativa, FUN: Fundación, SA: Sociedad Anónima, SL: Sociedad Limitada. 
ANEXO 3. EVOLUCIÓN DE LOS ESTADÍSTICOS PARA EL INCN DE LOS CEDE. FIGURA JURÍDICA

\begin{tabular}{|c|c|c|c|c|c|c|c|c|}
\hline & & & & & & & \\
\hline & & 2007 & 2008 & 2009 & 2010 & 2011 & 2012 & 2013 \\
\hline \multirow[b]{5}{*}{ ASO } & Media & 534.720 & 601.512 & 681.423 & 915.098 & 806.690 & 745.282 & 1.088 .430 \\
\hline & $\begin{array}{c}\text { Desviación } \\
\text { típica }\end{array}$ & 792.906 & 889.300 & 845.973 & 1.249 .867 & 880.979 & 699.109 & 1.064 .985 \\
\hline & $\begin{array}{c}\text { Percentil } \\
75 \\
\end{array}$ & 737.978 & 703.830 & 870.233 & 1.079 .842 & 1.005 .698 & 1.002 .570 & 1.168 .879 \\
\hline & Mediana & 149.628 & 225.383 & 373.318 & 346.760 & 565.092 & 598.290 & 718.929 \\
\hline & $\begin{array}{c}\text { Percentil } \\
25 \\
\end{array}$ & 50.631 & 48.845 & 76.398 & 118.535 & 202.563 & 209.458 & 549.232 \\
\hline \multirow[b]{5}{*}{$\mathrm{COOP}$} & Media & & & & 75.441 & 87.168 & 216.638 & 151.042 \\
\hline & $\begin{array}{c}\text { Desviación } \\
\text { típica }\end{array}$ & & & & & & & \\
\hline & $\begin{array}{c}\text { Percentil } \\
75 \\
\end{array}$ & & & & 75.441 & 87.168 & 216.638 & 151.042 \\
\hline & Mediana & & & & 75.441 & 87.168 & 216.638 & 151.042 \\
\hline & $\begin{array}{c}\text { Percentil } \\
25 \\
\end{array}$ & & & & 75.441 & 87.168 & 216.638 & 151.042 \\
\hline \multirow[b]{5}{*}{ FUN } & Media & 4.592 .663 & 3.580 .312 & 5.340 .546 & 5.999 .204 & 5.745 .649 & 4.334 .617 & 6.066 .139 \\
\hline & $\begin{array}{c}\text { Desviación } \\
\text { típica }\end{array}$ & 6.323 .017 & 5.345 .583 & 8.568 .264 & 9.278 .922 & 8.697 .098 & 5.997 .414 & 8.874 .077 \\
\hline & $\begin{array}{c}\text { Percentil } \\
75 \\
\end{array}$ & 9.063 .711 & 9.724 .745 & 15.223 .566 & 16.706.431 & 15.761 .312 & 11.236 .866 & 16.267 .638 \\
\hline & Mediana & 4.592 .663 & 1.017 .705 & 798.530 & 984.502 & 1.373 .721 & 1.371 .547 & 1.799 .843 \\
\hline & $\begin{array}{c}\text { Percentil } \\
25 \\
\end{array}$ & 121.615 & -1.515 & -458 & 306.679 & 101.913 & 395.439 & 130.936 \\
\hline \multirow[b]{5}{*}{$\begin{array}{l}\text { PERSONA } \\
\text { FISICA } \\
\end{array}$} & Media & 188.891 & 201.732 & 168.793 & 276.828 & 202.083 & 62.696 & 66.089 \\
\hline & $\begin{array}{c}\text { Desviación } \\
\text { típica }\end{array}$ & 198.378 & 195.797 & 149.695 & 313.388 & 231.888 & 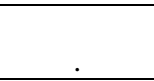 & 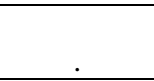 \\
\hline & $\begin{array}{c}\text { Percentil } \\
75 \\
\end{array}$ & 329.165 & 340.181 & 274.644 & 498.426 & 366.053 & 62.696 & 66.089 \\
\hline & Mediana & 188.891 & 201.732 & 168.793 & 276.828 & 202.083 & 62.696 & 66.089 \\
\hline & $\begin{array}{c}\text { Percentil } \\
25 \\
\end{array}$ & 48.616 & 63.283 & 62.943 & 55.229 & 38.114 & 62.696 & 66.089 \\
\hline \multirow[b]{5}{*}{ SA } & Media & 4.118 .670 & 4.553 .522 & 4.625 .741 & 6.172 .204 & 8.718 .848 & 8.702 .639 & 14.855 .583 \\
\hline & $\begin{array}{c}\text { Desviación } \\
\text { típica }\end{array}$ & 5.886 .427 & 6.930 .167 & 7.821 .824 & 10.764 .519 & 17.111.504 & 19.831 .248 & 33.621 .675 \\
\hline & $\begin{array}{c}\text { Percentil } \\
75\end{array}$ & 12.200 .468 & 6.845 .432 & 2.498 .776 & 5.254 .684 & 7.135 .634 & 5.136 .088 & 4.157.847 \\
\hline & Mediana & 537.545 & 774.167 & 780.644 & 1.200 .628 & 854.870 & 549.601 & 1.341 .556 \\
\hline & $\begin{array}{c}\text { Percentil } \\
25 \\
\end{array}$ & 92.913 & 128.555 & 141.913 & 123.163 & 147.991 & 153.335 & 112.802 \\
\hline \multirow[b]{5}{*}{ SL } & Media & 474.504 & 500.903 & 501.866 & 768.580 & 903.055 & 797.968 & 1.045 .602 \\
\hline & $\begin{array}{c}\text { Desviación } \\
\text { típica }\end{array}$ & 951.078 & 1.011 .255 & 1.013 .091 & 1.730 .686 & 1.891 .968 & 1.610 .528 & 2.271 .689 \\
\hline & $\begin{array}{c}\text { Percentil } \\
75 \\
\end{array}$ & 462.829 & 446.413 & 498.864 & 722.008 & 802.558 & 774.559 & 806.551 \\
\hline & Mediana & 180.609 & 191.404 & 206.714 & 218.744 & 293.382 & 291.293 & 302.010 \\
\hline & $\begin{array}{l}\text { Percentil } \\
25\end{array}$ & 59.547 & 73.846 & 79.160 & 83.486 & 111.889 & 119.834 & 111.772 \\
\hline
\end{tabular}

Fuente: Elaboración propia a partir de cuenta de Pérdidas y Ganancias presentadas por los CEdE. Nota: ASO: Asociación, COOP: Cooperativa, FUN: Fundación, SA: Sociedad Anónima, SL: Sociedad Limitada. 
ANEXO 4: EVOLUCIÓN DE LOS ESTADÍSTICOS DEL BENEFICIO BRUTO POR PERSONALIDAD JURÍDICA PARA LOS CEDE DE CASTILLA Y LEÓN

\begin{tabular}{|c|c|c|c|c|c|c|c|c|}
\hline & & 2007 & 2008 & 2009 & 2010 & 2011 & 2012 & 2013 \\
\hline \multirow[b]{5}{*}{ ASO } & Media & 117.519 & 66.879 & 99.279 & 34.665 & 44.395 & -6.171 & 62.971 \\
\hline & $\begin{array}{l}\text { Desviación } \\
\text { típica }\end{array}$ & 119.886 & 110.186 & 143.733 & 294.209 & 142.445 & 142.193 & 186.598 \\
\hline & $\begin{array}{c}\text { Percentil } \\
75 \\
\end{array}$ & 207.720 & 100.976 & 190.913 & 101.133 & 99.878 & 43.316 & 35.831 \\
\hline & Mediana & 84.654 & 19.920 & 25.932 & 36.493 & -983 & -3.024 & 7.409 \\
\hline & $\begin{array}{l}\text { Percentil } \\
25\end{array}$ & 20.497 & 565 & 54 & -19.736 & -42.375 & -73.079 & 10 \\
\hline \multirow[b]{5}{*}{ COOP } & Media & & & & -4.480 & -65.297 & 6.152 & 30.979 \\
\hline & $\begin{array}{l}\text { Desviación } \\
\text { típica }\end{array}$ & & & & & ( & . & \\
\hline & $\begin{array}{c}\text { Percentil } \\
75\end{array}$ & & & & -4.480 & -65.297 & 6.152 & 30.979 \\
\hline & Mediana & & & & -4.480 & -65.297 & 6.152 & 30.979 \\
\hline & $\begin{array}{c}\text { Percentil } \\
25\end{array}$ & & & & -4.480 & -65.297 & 6.152 & 30.979 \\
\hline \multirow[b]{5}{*}{ FUN } & Media & 146.985 & 146.653 & 228.720 & 285.311 & 258.843 & 128.436 & 94.493 \\
\hline & $\begin{array}{c}\text { Desviación } \\
\text { típica }\end{array}$ & 409.403 & 334.880 & 399.998 & 477.529 & 427.797 & 210.013 & 117.753 \\
\hline & $\begin{array}{c}\text { Percentil } \\
75\end{array}$ & 436.477 & 529.921 & 690.596 & 836.551 & 752.578 & 370.632 & 223.183 \\
\hline & Mediana & 146.985 & -557 & -1.087 & 21.308 & 25.367 & 17.883 & 68.165 \\
\hline & $\begin{array}{c}\text { Percentil } \\
25 \\
\end{array}$ & -142.507 & -89.406 & -3.349 & -1.925 & -1.417 & -3.207 & -7.867 \\
\hline \multirow[b]{5}{*}{$\begin{array}{l}\text { PERSONA } \\
\text { FISICA } \\
\end{array}$} & Media & 8.059 & 27.069 & 17.348 & 52.067 & 15.604 & -20.588 & -17.890 \\
\hline & $\begin{array}{l}\text { Desviación } \\
\text { típica }\end{array}$ & 23.791 & 34.258 & 26.501 & 72.108 & 37.161 & . & . \\
\hline & $\begin{array}{c}\text { Percentil } \\
75 \\
\end{array}$ & 24.882 & 51.294 & 36.087 & 103.055 & 41.880 & -20.588 & -17.890 \\
\hline & Mediana & 8.059 & 27.069 & 17.348 & 52.067 & 15.604 & -20.588 & -17.890 \\
\hline & $\begin{array}{c}\text { Percentil } \\
25 \\
\end{array}$ & -8.763 & 2.845 & -1.391 & 1.079 & -10.673 & -20.588 & -17.890 \\
\hline \multirow[b]{5}{*}{ SA } & Media & 127.522 & 154.955 & 122.218 & 294.190 & 268.332 & 317.713 & 446.449 \\
\hline & $\begin{array}{l}\text { Desviación } \\
\text { típica }\end{array}$ & 450.068 & 397.715 & 207.183 & 646.455 & 511.818 & 633.371 & 856.416 \\
\hline & $\begin{array}{c}\text { Percentil } \\
75\end{array}$ & 37.532 & 50.538 & 146.860 & 315.442 & 341.806 & 453.541 & 832.242 \\
\hline & Mediana & 459 & 20.916 & 37.261 & 53.434 & 87.171 & 20.286 & 11.535 \\
\hline & $\begin{array}{c}\text { Percentil } \\
25 \\
\end{array}$ & -15.172 & 6.160 & 18.688 & 12.161 & 924 & -5.448 & 531 \\
\hline \multirow[b]{5}{*}{ SL } & Media & 10.012 & 2.905 & 5.834 & 13.970 & 8.683 & 17.039 & 76.932 \\
\hline & $\begin{array}{c}\text { Desviación } \\
\text { típica }\end{array}$ & 67.163 & 64.041 & 69.569 & 74.289 & 88.637 & 87.401 & 299.910 \\
\hline & $\begin{array}{c}\text { Percentil } \\
75 \\
\end{array}$ & 34.454 & 22.873 & 18.617 & 17.897 & 26.757 & 32.518 & 66.759 \\
\hline & Mediana & 7.384 & 2.459 & 3.235 & 2.620 & 2.975 & 5.021 & 15.087 \\
\hline & $\begin{array}{c}\text { Percentil } \\
25\end{array}$ & -5.989 & -2.392 & -3.465 & -7.151 & -8.626 & -10.237 & -1.021 \\
\hline
\end{tabular}

Fuente: Elaboración propia a partir de las Cuentas de Pérdidas y Ganancias que presentan los CEdE.

Nota: ASO: Asociación, COOP: Cooperativa, FUN: Fundación, SA: Sociedad Anónima, SL: Sociedad Limitada. 